\title{
Fortbildung
}

\section{BETREUUNG VON KINDERN MIT ACHONDROPLASIE}

Matthieu Thimmesch, Olivier Bouchain, Silvia Schröder, Adrienne Henrotaux, Frédéric Lebrun

Übersetzer: Rudolf Schläpfer

\section{Zusammenfassung}

Die Achondroplasie ist die häufigste genetische Skelettdysplasie und beruht auf einer Mutation des FGFR3-kodierenden Gens. Die Diagnose wird manchmal vorgeburtlich gestellt. Meistens wird die Achondroplasie aber bei der Geburt oder während den ersten Lebensmonaten aufgrund charakteristischer dysmorpher Zeichen, wie die proximale Verkürzung der oberen und unteren Extremitäten, vermutet. Komplikationen sind häufig und müssen frühzeitig erkannt werden. Die Patienten haben ein erhöhtes Risiko für obstruktive Schlafapnoen und plötzlichen Kindstod. Aus neurologischer Sicht besteht das Risiko, eine Stenose des Spinalkanals verbunden mit zentralen Apnoen und einen Hydrocephalus zu entwickeln. Orthopädische Komplikationen betreffen vor allem die Wirbelsäule und die Gelenke. Die Betreuung muss multidisziplinär erfolgen. Wir beschreiben in diesem Artikel einen klinischen Fall und anschliessend die gesamte Betreuung.

\section{Einführung}

Die Achondroplasie ist die häufigste Skelettmissbildung. Die Frequenz variiert von 1/25'000 bis 1/30'000 Geburten. Sie beruht auf einer Mutation im FGFR3-Gen, das den Rezeptor 3 des Fibroblastenwachstumsfaktors kodiert. Respiratorische, neurologische und orthopädische Komplikationen sind häufig, weshalb diese Kinder eine spezifische Betreuung benötigen ${ }^{11}$.

\section{Fall beschreibung}

Unser Patient wurde in der 34. SSW geboren. Kurz nach der Geburt treten im Schlaf Schnarchen und Apnoen auf. Eine erste Polysomnografie (PSG) wies 42 (länger als 4 Sekunden dauernde) Apnoen nach, was die Überwachung durch ein kardiorespiratorisches Heimmonitoring rechtfertigte.

Bei der klinischen Untersuchung mit drei Monaten lassen eine Kyphose, kurze Extremitäten sowie ein auffallendes Gesicht die Diagnose Achondroplasie vermuten. Die radiologische Abklärung zeigt eine Kyphose (Abbildung 1) und kurze Glieder. Die genetische Untersuchung bestätigt das Vorhandensein einer de novo Mutation p.Gly380Arg im FGFR3-Gen. Das zervikale CT-Scan zeigt eine Stenose des Foramen magnum mit einem engen Spinalkanal und Komprimierung des Rückenmarkes und Hirnstammes. Das MRT bestätigt die Verengung des Foramen magnum mit medullärer Komprimierung auf $3.6 \mathrm{~mm}$, während der a.p.-Durchmesser des Rückenmarkes unterhalb davon $5.1 \mathrm{~mm}$ misst ( $A b$ bildung 2). Zudem besteht eine Volumenzunahme der intrakraniellen Liquorräume, bei einem oberen sinokortikalen und interkortikalen interhemisphären $\mathrm{Ab}$ stand von ca. $10 \mathrm{~mm}$. Die Vorderhörner haben einen Durchmesser von über $15 \mathrm{~mm}$. Ein erster Eingriff zur Dekompression des kraniozervikalen Überganges fand im Alter von acht Monaten statt.

Im Verlauf des ersten Winters erlebte der Knabe zahlreiche respiratorische Komplikationen, wobei mehrere Episoden schwerer Dekompensation eine invasive Beatmung benötigten. Es wurde eine subglottische Stenose nachgewiesen, die zur Verschlimmerung der obstruktiven Atembehinderung führte und im Alter von 9 Monaten eine Lasertherapie erforderlich machte. Diese Massnahme musste mehrmals wiederholt werden, bis im Alter von 10 Monaten schliesslich eine Tracheotomie, kombiniert mit einer Adenotomie, durchgeführt werden musste. Der Verlauf war in der Folge günstig, die Polysomnografie (PSG) normalisierte sich. Es fanden regelmässige ORL-, neurochirurgische, neuropädiatrische sowie pädiatrisch-pneumologische Nachkontrollen statt. Es traten jedoch weiterhin Episoden respiratorischer Dekompensation auf. Mehrere

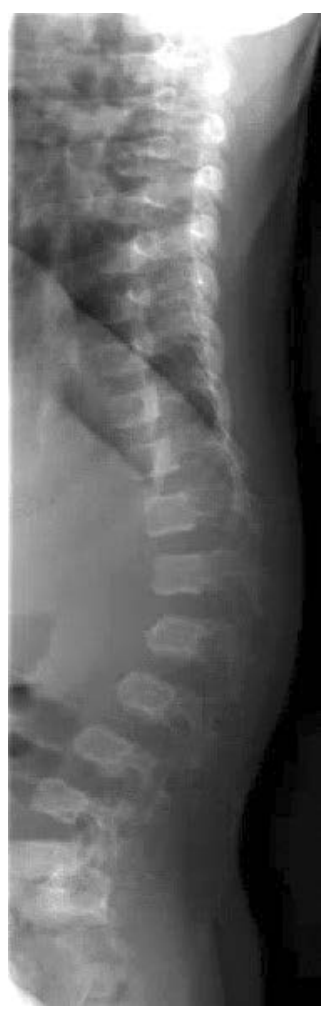

Abbildung 1: Röntgenaufnahme der Lendenwirbelsäule im Alter von 5 Monaten mit thorakolumbaler Kyphose.

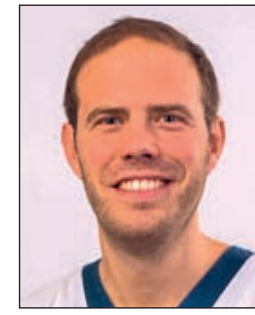

Matthieu Thimmesch 


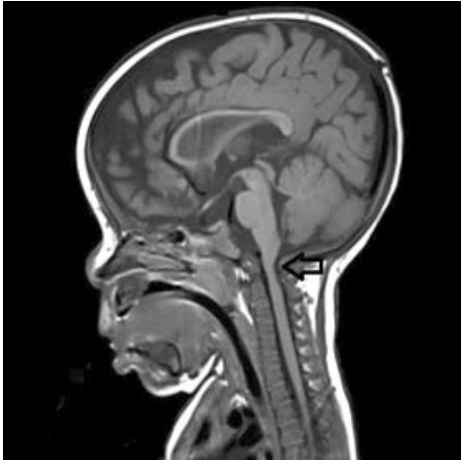

Abbildung 2: Hirn-MRT im Alter von 11 Monaten. Der sagittale T1-gewichtete Schnitt zeigt die Verengung des Foramen occipitale (Pfeil).

Versuche, das Tracheostoma bei gleichzeitiger Dilatation der subglottischen Stenose zu entfernen, misslangen.

Der Knabe konnte schliesslich im Alter von sechs Jahren von der Tracheotomie befreit werden. Es traten keine ernsthaften Atemwegskrisen mehr auf, doch kam es erneut zur Fragmentierung des Nachtschlafes mit Schnarchen und obstruktiven Apnoen. Die PSG wies zahlreiche obstruktive Apnoen mit einem Apnoe-Hypopnoe-Index von 31.3/Std. (normal <1.5/Std.) und einem obstruktiven Schlafapnoe-Index von 20.9/ Std. (normal <1/Std.) nach (Abbildung 3). Die Positivdruckbeatmung führt zu einer deutlichen Besserung der Schlafqualität und zur Normalisierung der PSG. Compliance und Toleranz sind vorzüglich.

Die neurologische Entwicklung zeichnet sich durch einen motorischen und sprachlichen Entwicklungsrückstand aus, verbunden mit einer leichten geistigen Entwicklungsstörung. Der Knabe wird heilpädagogisch betreut. Im Alter von acht Jahren musste er wegen eines Rezidivs der Stenose im Bereich des zervikalen Überganges erneut operiert werden. Bezüglich orthopädischer Komplikationen ist in naher Zukunft die chirurgische Korrektur der Beinachsen vorgesehen. Die Hyperlordose im Bereich der Lendenwirbelsäule wird vorläufig konservativ betreut.

\section{Diskussion}

Obwohl die Diagnose durch das Ausmessen kurzer Röhrenknochen bei der Ultraschalluntersuchung im dritten Trimenon schon in utero vermutet werden kann, wird die Diagnose Achondroplasie meist erst nach der Geburt und manchmal, wie bei unserem $\mathrm{Pa}$ tienten erst nach einigen Monaten gestellt. Folgende phänotypischen Merkmale sind die wichtigsten dieser Krankheit: Proximale Verkürzung der Extremitäten (Rhizomelie), dreizackförmige Hände mit kurzen Fingern, Makrozephalie mit vorgewölbter Stirn, flache $\mathrm{Nase}^{1)}$. Die Diagnose ist bei frühgeborenen Kindern erschwert ${ }^{2)}$. Die radiologische Abklärung offenbart abnorme Metaphysen, rhizomele Verkürzung der Extremitäten, eine relativ breite Schädelwölbung mit kleiner Schädelbasis, einen kleinen Beckenkamm, verengte Rippen, deformierte Wirbel mit verschmälertem Abstand der Bogenwurzeln ${ }^{3)}$.

Die Krankheit beruht auf einer Mutation im FGFR3-Gen. Zwei Mutationen (c.1138G $>$ A und c.1138G $>$ C) sind für $99 \%$ der Fälle verantwortlich ${ }^{4}$. Ist das Protein FGFR3 mutiert, wird es aktiv und hemmt das Wachstum der Chondrozyten und somit der Knochen ${ }^{5}$. Die mittlere Erwachsenengrösse beträgt für Männer $132 \mathrm{~cm}$ und für Frauen $124 \mathrm{~cm}$. Es sollten eigene Wachstumskurven für Kinder mit Achondroplasie benutzt werden ${ }^{6}$.

Diese Patienten haben ein erhöhtes Risiko für Atemstörungen während dem Schlaf, sowie für neurologische und orthopädische Komplikationen, weshalb eine frühzeitige Diagnose wichtig ist. Eine kürzlich erschienene Literaturreview gibt eine vollständige Übersicht der Betreuung von Patienten mit Achondroplasie ${ }^{1)}$.

\section{Atemstörungen während des Schlafes}

Schon kurz nach der Geburt besteht bei Kindern mit Achondroplasie ein erhöhtes Risiko für einen plötzlichen Kindstod durch das Auftreten von zentralen und obstruktiven Apnoen. Zentrale Apnoen sind bedingt durch die Komprimierung des Zervikanalkanals, obstruktive Apnoen durch die Gesichtsdysmorphie, die zu einer Verminderung des Volumens der oberen

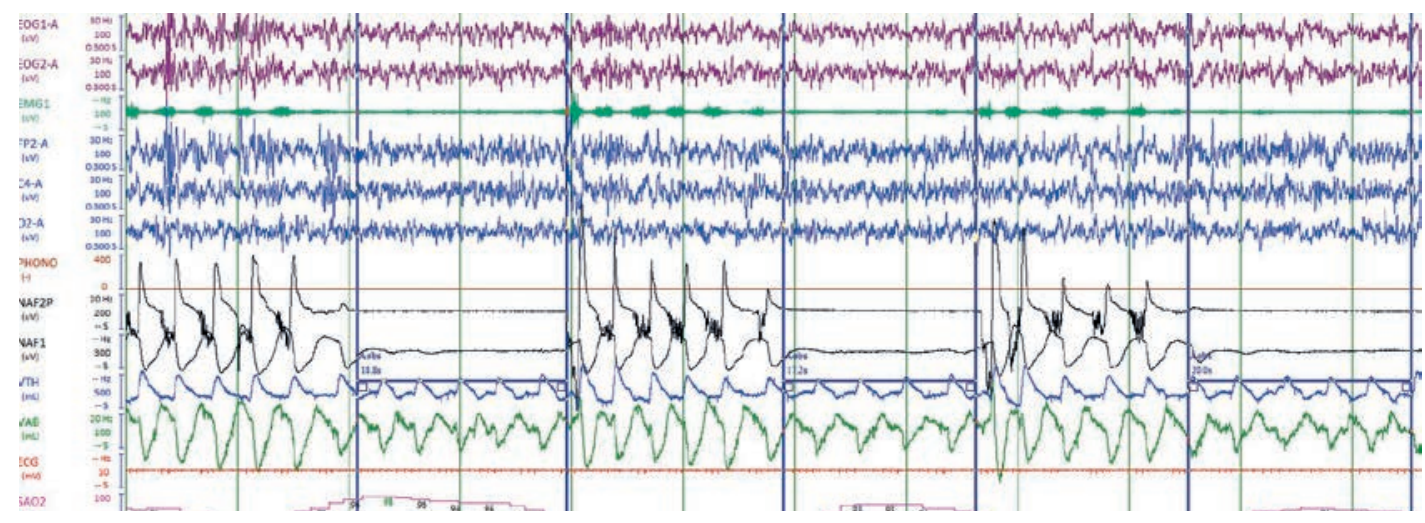

Abbildung 3: Polysomnografie im Alter von 7 Jahren mit zahlreichen obstruktiven Apnoen (Aobs). Unterbrechung des Atemflusses nasal (NAF2P) und mit Temperatursensoren gemessen (NAF1), bei erhaltenen Thorax- (VTH) und Abdominalbewegungen (VAB). Obstruktive Apnoen assoziiert mit Sauerstoffentsättigung. 


\section{Fortbildung}

Luftwege führt ${ }^{7}$ ). Es ist deshalb wichtig, polysomnographische Kontrollen im Schlaf durchzuführen und den Eltern eventuell ein kardiorespiratorisches Heimmonitoring vorzuschlagen, wie dies bei unserem $\mathrm{Pa}$ tienten der Fall war. Ebenso soll frühzeitig eine zerebrale Bildgebung (MRT) durchgeführt werden.

In der Folge können sich die obstruktiven Apnoen verschlimmern und in einem Drittel der Fälle zu einem obstruktiven Schlafapnoe-Syndrom (OSAS) führen. Schnarchen ist kein klinisch zuverlässiges Symptom, da die engen Atemwege beim Einatmen zu Turbulenzen führen. Es ist deshalb wichtig, bei der Anamnese nach weiteren Zeichen eines OSAS zu suchen, wie der Eindruck von Apnoen mit vermehrter inspiratorischer Anstrengung und Hyperextension des Nackens, morgendliche Kopfschmerzen, häufiges nächtliches Aufwachen, sekundäre Enuresis, Reizbarkeit (häufiger bei jüngeren Kindern), Müdigkeit tagsüber oder Lernschwierigkeiten beim älteren Kind ${ }^{1,8)}$. Das OSAS kann durch Hypertrophie der Gaumenund/oder Rachenmandeln, gastroösophageale Refluxkrankheit, zentrale Apnoen durch Kompression des zervikalen Überganges sowie Adipositas verstärkt werden. Bei unserem Patienten wurde das OSAS durch die subglottische Stenose, die keine übliche Komplikation der Achondroplasie darstellt, verstärkt. Die Diagnose beruht auf der Polysomnografie verbunden mit transkutaner PCO-Messung. Werden die obstruktiven Apnoen nicht behandelt, führt dies zu verminderter Schlafqualität und damit zu einer Störung der Wachstumshormonsekretion, was die Wachstumsprognose verschlechtern kann ${ }^{9}$. Im Erwachsenenalter vergrössert das OSAS das Risiko kardiovaskulärer Pathologien, wie dies auch bei Patienten ohne Achondroplasie der Fall ist ${ }^{1,10)}$. Das OSAS wird durch Übergewicht, was bei Patienten mit Achondroplasie häufig ist, verschlimmert. Die Behandlung besteht in der Entfernung der Adenoide mit oder ohne Tonsillektomie. Leider ist die ORL-chirurgische Behandlung nur in gewissen Fällen wirksam und es wird oft eine nicht-invasive nächtliche Beatmung notwendig8).

Die Atemstörung kann manchmal auch auf einer restriktiven Ventilationsstörung beruhen, bedingt durch ein kleines Thoraxvolumen, übermässige Compliance und assoziierte Verformungen des Brustkorbes. In extremen Fällen kann es zu chronischer Hypoxämie mit klinischen Atemnotzeichen und einem Abflachen der Wachstumskurve kommen. Es wird in diesen Fällen empfohlen, eine nächtliche Oxymetrie, eventuell verbunden mit transkutaner PCO-Messung durchzuführen. Es kann in gewissen Fällen notwendig sein, Sauerstoff zu verabreichen, und in sehr schweren Fällen vorübergehend eine Tracheostomie anzulegen ${ }^{1)}$. Im Falle unseres Patienten erforderte die subglottische Stenose die Tracheotomie.

\section{Neurologische Störungen}

Wie schon erwähnt, kann der verminderte Durchmesser des Foramen ovale, bedingt durch das eingeschränkte Knorpelwachstum der Schädelbasis, zu einer Komprimierung des Überganges Hirnstamm-Rü- ckenmark und der Arteriae vertebrales führen, was wiederum das Risiko eines plötzlichen Kindstodes erhöht. Bei der neurologischen Untersuchung des achondroplasischen Säuglings ist auf Zeichen pyramidaler Reizung (Hyperreflexie mit Ausweitung der reflexogenen Zone, Klonus, Babinski- und Hoffman-Zeichen) zu achten. Die Untersuchung ist zeitnah durch ein MRT der Schädelbasis und, wie oben erwähnt, eine Polysomnografie zu ergänzen. Etwa 10\% der Säuglinge benötigen eine chirurgische Dekompression des kraniozervikalen Überganges ${ }^{1}$. Die Indikation für einen chirurgischen Eingriff ist in erster Linie durch die Klinik sowie Zeichen von Druck auf das Knochenmark im MRT (hyperintenses Signal bei T2-Gewichtung) gegeben ${ }^{10)}$.

Das Risiko des plötzlichen Kindstodes nimmt nach dem ersten Lebensjahr ab, doch das Kompressionsrisiko des Rückenmarkes besteht weiter, weshalb regelmässige neuropädiatrische Kontrollen wichtig sind, um verdächtige Zeichen zu erfassen. Es ist nicht selten, dass wie bei unserem Patienten ein zweiter Eingriff notwendig wird.

Kinder mit Achondroplasie haben ebenfalls ein erhöhtes Risiko, einen Hydrozephalus zu entwickeln und bei $4.3 \%$ wird eine ventrikulo-peritoneale Drainage notwendigin). Bei Patienten mit Achondroplasie einen Hydrozephalus festzustellen ist nicht immer einfach, da die meisten dieser Kinder eine nicht pathologische Makrozephalie mit Ventrikulomegalie und überschüssigem Liquor aufweisen. Die regelmässige Messung des Kopfumfanges bis ins Alter von 5-6 Jahren ist deshalb wichtig'). Hirndrucksymptome treten meist schleichend und unmerklich auf.

Die kognitive Entwicklung ist bei Kindern mit Achondroplasie meist normal. Ein Entwicklungsrückstand kann jedoch als Folge eines schweren OSAS auftreten und eine heilpädagogische Betreuung erfordern ${ }^{12}$. Ebenso können epileptische Anfälle auftreten, die durch eine schlechte Schlafqualität verschlimmert werden können ${ }^{1}$. Die Missbildungen der Wirbelsäule werden im Kapitel orthopädische Komplikationen beschrieben.

\section{Orthopädische Komplikationen}

Kinder mit Achondroplasie leiden an zahlreichen orthopädischen Komplikationen der Wirbelsäule und der oberen und unteren Extremitäten. Beinahe alle Kinder entwickeln im Verlaufe des ersten Lebensjahres eine thorakolumbale Kyphose ${ }^{13}$. Diese Kyphose ist meist reversibel, kann aber in 10-15\% der Fälle bis ins Erwachsenenalter fortbestehen. Einige einfache Ratschläge, wie kein Sitzen ohne Rückenlehne und auf den Babywalker verzichten, tragen dazu bei, das Andauern der Kyphose zu vermeiden ${ }^{1}$.

Beim Erlernen des freien Gehen entwickeln zahlreiche Kinder eine Hyperlordose, die oft die Eltern beunruhigt, jedoch meist asymptomatisch ist und keiner Behandlung bedarf. Ist die Lordose ausgeprägter, besteht beim Jugendlichen oder Erwachsenen ein erhöhtes Risiko für das intermittierende Auftreten einer Claudicatio spinalis und einer Spinalkanalstenose. Die 


\section{Fortbildung}

physiotherapeutische Behandlung kann die Ausprägung der Hyperlordose mindern ${ }^{1)}$.

Bei allen Patienten mit Achondroplasie ist das Kaliber des gesamten Wirbelkanals vermindert. Symptome treten meist erst nach der Adoleszenz, im Mittel um die 40 Jahre auf, in Form einer Claudicatio die den Aktionsradius einschränkt. Bei leichteren Fällen kann eine konservative Behandlung, bestehend aus Gewichtsabnahme, Physiotherapie und einem Programm gezielter Übungen, um der Hyperlordose entgegenzuwirken, die Symptome lindern. Bei ausgeprägter lumbosakraler Spinalkanalstenose mit Gehstörung, Ermüdbarkeit der Beinmuskulatur und neurologischer Blase, führt die klassische Behandlung, bestehend aus einer breiten und ausgedehnten dorsalen Laminektomie, zur Besserung der Symptome ${ }^{1,14)}$.

An den unteren Extremitäten fällt ab dem Alter von zwei Jahren die zunehmende Bildung eines Genu varum auf. Diese Missbildung tritt bei 30-50\% der Patienten auf, wovon ein Viertel eine chirurgische Korrektur benötigen wird. Sie kommt häufiger bei Knaben vor, ist oft asymmetrisch und begünstigt das Auftreten einer Osteoarthritis. Die zusätzliche Hypermobilität der Knie und das Genu recurvatum verschlimmern die Symptome und Schmerzen. An den oberen Extremitäten fällt bei beinahe allen Patienten eine Hypermobilität der Hand- und Schultergelenke auf, während die Ellbogen im Gegenteil eher versteift sind. Bei unserem Patienten ist demnächst eine Operation der unteren Extremitäten vorgesehen ${ }^{1)}$.

\section{Weitere Störungen}

Patienten mit Achondroplasie haben sehr oft eine Malokklusion des Kiefers, bedingt durch die Maxillahypoplasie und ein verstärktes Wachstum des Unterkiefers. Es besteht ebenfalls ein erhöhtes Risiko für kardiovaskuläre Pathologien ${ }^{1)}$.

\section{Fazit}

Die Achondroplasie betrifft zahlreiche Organsysteme, deren Folgen sich bereits bei der Geburt bemerkbar machen können. Die Inzidenz des plötzlichen Kindstodes ist erhöht. Bezüglich respiratorischer Symptome ist es wichtig, ein OSAS frühzeitig zu erkennen, um kognitive Defizite zu verhindern. In seltenen Fällen, wie bei unserem Patienten, kann eine Tracheotomie notwendig werden. Bezüglich Neurostatus muss regelmässig nach Zeichen einer Spinalkompression gesucht und der Kopfumfang gemessen werden. Der Bewegungsapparat kann ebenfalls Ursache zahlreicher Komplikationen sein. Die frühzeitige Diagnose in der Neugeborenenperiode und eine multidisziplinäre Betreuung sind wesentlich, um die Prognose und Lebensqualität dieser Patienten zu verbessern.

\section{Referenzen}

1) Pauli RM. - Achondroplasia: a comprehensive clinical review. Orphanet J Rare Dis. 2019 3;14(1):1

2) Fagen KE, Blask AR, Rubio El, Bulas DI. - Achondroplasia in the Premature Infant: An Elusive Diagnosis in the Neonatal Intensive Care Unit. AJP Rep. 2017;7(1):e8-e12.

3) Pereira E. - Achondroplasia. Pediatr Rev. 2019;40(6):316-318.

4) Pauli RM, Legare JM. Achondroplasia. In: Adam MP, Ardinger HH, Pagon RA, Wallace SE, Bean LJH, Stephens K, Amemiya A, editors. GeneReviews', 2018.

5) Webster MK, Donoghue DJ. Constitutive activation of fibroblast growth factor receptor 3 by the transmembrane domain point mutation found in achondroplasia. EMBO J. 1996 1;15(3):520-7.

6) Merker A, Neumeyer L, Hertel NT, Grigelioniene G, Mäkitie O, Mohnike $\mathrm{K}$ et al. - Growth in achondroplasia: Development of height, weight, head circumference, and body mass index in a European cohort. Am J Med Genet A. 2018;176(8):1723-1734.

7) Pauli RM, Scott Cl, Wassman ER, Gilbert EF, Leavitt LA, Ver Hoeve J, et al. - Apnea and sudden unexpected death in infants with achondroplasia. J Pediatr. 1984;104(3):342-8.

8) Tenconi R, Khirani S, Amaddeo A, Michot C, Baujat G, Couloigne V. - Sleep-disordered breathing and its management in children with achondroplasia. Am J Med Genet A. 2017;173(4):868-878

9) Lanfranco F, Motta G, Minetto MA, Ghigo E, Maccario M. Growth hormone/insulin-like growth factor-l axis in obstructive sleep apnea syndrome: an update. J Endocrinol Invest. 2010;33(3):192-6.

10) White KK, Parnell SE, Kifle Y, Blackledge M, Bompadre V. - Is there a correlation between sleep disordered breathing and foramen magnum stenosis in children with achondroplasia? Am J Med Genet A. 2016;170A(1):32-41.

11) King JA, Vachhrajani S, Drake JM, Rutka JT. - Neurosurgical implications of achondroplasia. J Neurosurg Pediatr. 2009;4(4):297-306.

12) Afsharpaiman $S^{1}$, Sillence DO, Sheikhvatan M, Ault JE, Waters K. Respiratory events and obstructive sleep apnea in children with achondroplasia: investigation and treatment outcomes. Sleep Breath. 2011;15(4):755-61.

13) Pauli RM, Breed A, Horton VK, Glinski LP, Reiser CA. Prevention of fixed, angular kyphosis in achondroplasia. J Pediatr Orthop. 1997;17(6):726-33.

14) Pyeritz RE, Sack GH Jr, Udvarhelyi GB. - Thoracolumbosacral laminectomy in achondroplasia: long-term results in 22 patients. Am J Med Genet. 1987;28(2):433-44.

\footnotetext{
Autoren

Dr. med. Matthieu Thimmesch, Service de pédiatrie, Centre Hospitalier Chrétien, clinique du MontLégia, Liège, Belgique

Olivier Bouchain, Service d'ORL, Centre Hospitalier Universitaire de Liège, Belgique

Silvia Schröder, Service d'orthopédie pédiatrique, Centre Hospitalier Chrétien, clinique du MontLégia, Liège, Belgique

Adrienne Henrotaux, Service de neurochirurgie, Centre Hospitalier Chrétien, clinique du MontLégia, Liège, Belgique

Frédéric Lebrun, Service de pédiatrie \& soins intensifs pédiatriques, Centre Hospitalier Chrétien, clinique du MontLégia, Liège, Belgique
}

Die Autoren haben keine finanziellen oder persönlichen Verbindungen im Zusammenhang mit diesem Beitrag deklariert. 